\title{
On the Existence, Uniqueness and Application of the
}

\section{Finite Difference Method for Solving Robin Elliptic Boundary Value Problem}

\author{
Germain Nguimbi ${ }^{1}$, Diogène Vianney Pongui Ngoma $^{1}$, Vital Delmas Mabonzo ${ }^{2}$, Bienaime Bervi Bamvi Madzou ${ }^{3} \&$ \\ Melchior Josièrne Jupy Kokolo ${ }^{2}$ \\ ${ }^{1}$ Ecole Nationale Superieure Polytechnique, Marien Ngouabi University, Brazzaville, Congo \\ ${ }^{2}$ Ecole Normale Superieure, Marien Ngouabi University, Brazzaville, Congo \\ ${ }^{3}$ Faculty of Sciences and Technics, Marien Ngouabi University, Brazzaville, Congo \\ Correspondence: Diogène Vianney Pongui Ngoma, Ecole Nationale Superieure Polytechnique, Marien Ngouabi Univer- \\ sity, Brazzaville, Congo.
}

Received: October 29, 2018 Accepted: November 20, 2018 Online Published: January 14, 2019

doi:10.5539/jmr.v11n1p26

URL: https://doi.org/10.5539/jmr.v11n1p26

\begin{abstract}
This paper refers to mathematical modelling and numerical analysis. The analysis to be presented through this paper deals with Robin's problem which boundary equation is a linear combination of Dirichlet and Neumann-type boundary conditions. For this purpose we proved the existence and uniqueness of the solution. It is worth noting that the implementation of numerical simulations depends on the type of problem since it requires a search for explicit solution. Consequently, the motivation exists in this paper for choosing a classical method of variation of constants and employing a finite difference method to find the exact and numerical solutions, respectively so that numerical simulations were implemented in $S c i l a b$.
\end{abstract}

Keywords: Robin problem, finite difference method, numerical simulations

\section{Introduction}

Let $\Omega$ be a bounded domain in $\mathbb{R}^{N}, N \geqslant 1$, with boundary $\partial \Omega$. We shall consider the following Robin elliptic boundary value problem (H. Beghr \& G. Harutjunjan, 2006)

$$
\left\{\begin{array}{l}
-\Delta u(x)+c(x) u(x)=f(x), \quad x \in \Omega, \\
\alpha u(x)+\frac{\partial u(x)}{\partial \eta}=g(x), x \in \partial \Omega,
\end{array}\right.
$$

where $u$ is the solution of problem, $f \in L^{2}(\Omega), g \in L^{2}(\partial \Omega), \eta \quad$ is the exterior normal to the boundary $\partial \Omega$ of the domain $\Omega$. We shall assume that $c(x) \geqslant c_{o}>0$ and $\alpha \in \mathbb{R}_{+}$.

Note that, by combining the Dirichlet- and Neumann-type boundary conditions we get a Robin-type boundary condition. Then for example, if $\alpha=0$ the problem (1) becomes Neumann boundary problem. Finite element method and finite difference method for solving Dirichlet and Neumann problems have been extensively analyzed by (Gabriel et al., 2015), ( Bourchra Bensiali et al., 2006), (H. Beghr \& G. Harutjunjan, 2006) and (Aslak Tveito \& Ragnar Winther, 1998). The resolution algorithm and the implementation of numerical simulations depend on the type of problem and require a search of explicit solution. Then, the aim of this paper is to design and employ a finite difference method for approximating the solution of (1). It is also appropriate to choose a classical method of variation of constants for the one-dimensional Robin problem to be solved exactly. Thus using the above exact and numerical solutions, numerical simulations will be implemented in Scilab. The analysis to be presented below makes strong use of the results and arguments of (Shao-Gao Deng, 2009) and ( M. Hinze et al., 2009). An outline of this paper is as follows. A weak form will be presented. The existence and uniqueness of the solution of the problem (1) will be demonstrated. Then, the problem will be solved numerically and analytically by using the finite difference method and the classical method of variation of constants, respectively. Finally using these solutions, numerical simulation will be implemented in $S$ cilab.

\section{Weak Form of the Problem}

For later use, we seek the Sobolev space containing the solution $u$

$$
f \in L^{2}(\Omega) \Longrightarrow-\Delta u(x)+c(x) u(x) \in L^{2}(\Omega),
$$




$$
\begin{aligned}
& \Delta u(x) \in L^{2}(\Omega), \\
& u \in H^{1}(\Omega),
\end{aligned}
$$

So that

$$
V=H^{1}(\Omega) \text {, which is a Hilbert space (Peter Knabner et al., 2000). }
$$

In the following we multiply the first equation of (1) by $v$, integrate over $\Omega$ to obtain

$$
\int_{\Omega}-\Delta u . v d \Omega+\int_{\Omega} c . u d \Omega=\int_{\Omega} f . v d \Omega, \quad \text { for } \quad v \in H^{1}(\Omega)
$$

By using Green's formula (M. Hinze et al., 2009), we have

$$
\begin{gathered}
\int_{\Omega} \nabla u \cdot \nabla v d \Omega-\int_{\partial \Omega} \frac{\partial u}{\partial \eta} \cdot v d \sigma+\int_{\Omega} c \cdot u \cdot v d \Omega=\int_{\Omega} f \cdot v d \Omega \\
\int_{\Omega} \nabla u \cdot \nabla v d \Omega-\int_{\partial \Omega}(g-\alpha u) \cdot v d \sigma+\int_{\Omega} c \cdot u \cdot v d \Omega=\int_{\Omega} f \cdot v d \Omega, \\
\int_{\Omega} \nabla u \cdot \nabla v d \Omega-\int_{\partial \Omega} g v d \sigma+\alpha \int_{\partial \Omega} u v d \sigma+\int_{\Omega} c \cdot u \cdot v d \Omega=\int_{\Omega} f \cdot v d \Omega, \\
\int_{\Omega} \nabla u \cdot \nabla v d \Omega+\alpha \int_{\partial \Omega} u \cdot v d \sigma+\int_{\Omega} c \cdot u \cdot v d \Omega
\end{gathered}
$$

Hence the variational formulation of (1) may be written

$$
a(u, v)=L(v) \quad \text { for any } \quad v \in H^{1}(\Omega),
$$

Where

$$
a(u, v)=\int_{\Omega} \nabla u \cdot \nabla v d \Omega+\alpha \int_{\partial \Omega} u \cdot v d \sigma+\int_{\Omega} c . u \cdot v d \Omega, \quad L(v)=\int_{\partial \Omega} g . v d \sigma+\int_{\Omega} f \cdot v d \Omega .
$$

\section{Existence and Uniqueness of the Solution}

The demonstration of the existence and uniqueness of the solution of the problem (1) relies on Lax-Milgram theorem (M. Hinze et al., 2009). Since, it is trivial that $a$ is bilinear, it remains to prove the continuity of $a$.

We have

$$
\begin{aligned}
|a(u, v)| & =\left|\int_{\Omega} \nabla u \cdot \nabla v d \Omega+\alpha \int_{\partial \Omega} u \cdot v d \sigma+\int_{\Omega} c \cdot u \cdot v d \Omega\right| \\
& \leqslant \int_{\Omega}|\nabla u \cdot \nabla v| d \Omega+|\alpha| \int_{\partial \Omega}|u \cdot v| d \sigma+\int_{\Omega}|c \cdot u \cdot v| d \Omega
\end{aligned}
$$

Thus

$$
|a(u, v)| \leqslant \int_{\Omega}|\nabla u . \nabla v| d \Omega+|\alpha| \int_{\partial \Omega}|u . v| d \sigma+|c| \int_{\Omega}|u . v| d \Omega
$$

By using Cauchy-Schwartz's inequality, we obtain

$$
\begin{gathered}
|a(u, v)| \leqslant\left(\int_{\Omega}|\nabla u|^{2} d \Omega\right)^{\frac{1}{2}}\left(\int_{\Omega}|\nabla u|^{2} d \Omega\right)^{\frac{1}{2}}+|\alpha|\left(\int_{\partial \Omega}|u|^{2} d \sigma\right)^{\frac{1}{2}}\left(\int_{\partial \Omega}|v|^{2} d \sigma\right)^{\frac{1}{2}}+|c|\left(\int_{\Omega}|u|^{2} d \Omega\right)^{\frac{1}{2}}\left(\int_{\Omega}|v|^{2} d \Omega\right)^{\frac{1}{2}}, \\
\leqslant\|\nabla u\|_{L^{2}(\Omega)}\|\nabla v\|_{L^{2}(\Omega)}+|\alpha|\|u\|_{L^{2}(\partial \Omega)}\|v\|_{L^{2}(\partial \Omega)}+|c|\|u\|_{L^{2}(\Omega)}\|v\|_{L^{2}(\Omega)} \\
|a(u, v)| \leqslant\|u\|_{H^{1}(\Omega)}\|v\|_{H^{1}(\Omega)}+|\alpha|\|u\|_{L^{2}(\partial \Omega)}\|v\|_{L^{2}(\partial \Omega)}+|c|\|u\|_{L^{2}(\Omega)}\|v\|_{L^{2}(\Omega)}
\end{gathered}
$$


By using Poincare's inequality and the trace inequality (Aslak et al., 1998), we hence obtain

$$
\begin{gathered}
|a(u, v)| \leqslant\|u\|_{H^{1}(\Omega)}\|v\|_{H^{1}(\Omega)}+k_{3} k_{4}|\alpha|\|u\|_{H^{1}(\Omega)}\|v\|_{H^{1}(\Omega)}+|c| k_{1} k_{2}\|u\|_{H^{1}(\Omega)}\|v\|_{H^{1}(\Omega)}, \\
\leqslant\left(1+k_{3} k_{4}|\alpha|+|c| k_{1} k_{2}\right)\|u\|_{H^{1}(\Omega)}\|v\|_{H^{1}(\Omega)} ; \\
|a(u, v)| \leqslant k\|u\|_{H^{1}(\Omega)}\|v\|_{H^{1}(\Omega)} .
\end{gathered}
$$

where

$$
k=1+k_{3} k_{4}|\alpha|+|c| k_{1} k_{2}
$$

Therefore $a$ is continuous.

Since obviously, $L$ is linear, then it remains to prove that $L$ is continuous.

$$
\begin{aligned}
|L(v)| & =\left|\int_{\partial \Omega} g \cdot v d \sigma+\int_{\Omega} f \cdot v d \Omega\right| \\
& \leqslant\left|\int_{\partial \Omega} g \cdot v d \sigma\right|+\left|\int_{\Omega} f \cdot v d \Omega\right| \\
& \leqslant \int_{\partial \Omega}|g \cdot v| d \sigma+\int_{\Omega}|f \cdot v| d \Omega .
\end{aligned}
$$

Then, it follows from Cauchy-Schwartz's inequality (M. Hinze et al., 2009)

$$
\begin{aligned}
& \left.|L(v)| \leqslant\left(\int_{\partial \Omega}|g|^{2} d \sigma\right)^{\frac{1}{2}}\left(\int_{\partial \Omega}|v|^{2} d \sigma\right)^{\frac{1}{2}}\right)+\left(\int_{\Omega}|f|^{2} d \Omega\right)^{\frac{1}{2}}\left(\int_{\Omega}|v|^{2} d \Omega\right)^{\frac{1}{2}}, \\
& |L(v)| \leqslant\|g\|_{L^{2}(\partial \Omega)}\|v\|_{L^{2}(\partial \Omega)}+\|f\|_{L^{2}(\Omega)}\|v\|_{L^{2}(\Omega)},
\end{aligned}
$$

By Poincare inequality we have

$$
\|v\|_{L^{2}(\Omega)} \leqslant M_{1}\|v\|_{H^{1}(\Omega)} .
$$

Moreover, according to the continuity of the trace function on $H^{1}(\Omega),\left.v \longmapsto v\right|_{\partial \Omega}=v$ such that:

$$
\|v\|_{L^{2}(\partial \Omega)} \leqslant M_{2}\|v\|_{H^{1}(\Omega)}
$$

and the following known results (Aslak et al., 1998)

$$
\begin{aligned}
f \in L^{2}(\Omega) & \Longrightarrow\|f\|_{L^{2}(\Omega)} \leqslant M_{3} \\
g \in L^{2}(\partial \Omega) & \Longrightarrow\|g\|_{L^{2}(\partial \Omega)} \leqslant M_{4}
\end{aligned}
$$

Thus

$$
\begin{gathered}
|L(v)| \leqslant M_{2} M_{4}\|v\|_{H^{1}(\Omega)}+M_{1} M_{3}\|v\|_{H^{1}(\Omega)} ; \\
\leqslant\left(M_{2} M_{4}+M_{1} M_{3}\right)\|v\|_{H^{1}(\Omega)} ; \\
|L(v)| \leqslant M\|v\|_{H^{1}(\Omega)}, \quad \text { where } \quad M=M_{2} M_{4}+M_{1} M_{3}
\end{gathered}
$$

Hence the linear form $L$ is continuous.

Next, let us prove the coercitivity of bilinear form $a$.

Set $u=v$, then

$$
a(u, u)=\int_{\Omega} \nabla u \nabla u d \Omega+\alpha \int_{\partial \Omega} u \cdot u d \sigma+\int_{\Omega} c \cdot u \cdot u d \Omega
$$




$$
\begin{aligned}
& a(u, u) \geqslant \int_{\Omega} \nabla u^{2} d \Omega+c_{0} \int_{\Omega} u^{2} d \Omega+\alpha \int_{\partial \Omega} u^{2} d \sigma \\
& a(u, u) \geqslant\|\nabla u\|_{L^{2}(\Omega)}^{2}+c_{o}\|u\|_{L^{2}(\Omega)}^{2}+\alpha\|u\|_{L^{2}(\partial \Omega)}^{2} \geqslant\|\nabla u\|_{L^{2}(\Omega)}^{2}+c_{0}\|u\|_{L^{2}(\Omega)}^{2} ; \\
& a(u, u) \geqslant \min \left(1, c_{0}\right)\|\nabla u\|_{L^{2}(\Omega)}^{2}+\min \left(1, c_{0}\right)\|u\|_{L^{2}(\Omega)}^{2}, \\
& a(u, u) \geqslant \min \left(1, c_{0}\right)\left(\|\nabla u\|_{L^{2}(\Omega)}^{2}+\|u\|_{L^{2}(\Omega)}^{2}\right), \\
& \geqslant \min \left(1, c_{0}\right)\|u\|_{L^{2}(\Omega)}^{2}
\end{aligned}
$$

Let

$$
\gamma=\min \left(1, c_{0}\right)
$$

Then

Hence $a$ is coercive.

$$
a(u, u) \geqslant \gamma\|u\|_{H^{1}(\Omega)}^{2} .
$$

Then, from the Lax-Milgram theorem, it follows that problem (1) has a unique solution $u \in H^{1}(\Omega)$ such that $a(u, v)=$ $L(v)$ and $\|u\|_{H^{1}(\Omega)} \leqslant \frac{\|L\|}{\gamma}$.

\section{Numerical Resolution of the Problem}

In this section, we shall consdier the finite difference method for solving the one-dimensional Robin's problem (Loredana Lanzani et al., 2006) which is as follows:

$$
\left\{\begin{array}{l}
\left.-u^{\prime \prime}(x)+c(x) u(x)=f(x), x \in\right] 0,1[ \\
u^{\prime}(0)+\alpha u(0)=g(0), u^{\prime}(1)+\alpha u(1)=g(1), \alpha>0
\end{array}\right.
$$

We then consider a partition

$$
0=x_{0}<x_{1}<\ldots<x_{N}<x_{N+1}=1,
$$

of the interval $[0,1]$, where $N \in \mathbb{N}$. Suppose the uniform step be given by $h_{i}=x_{i+1}-x_{i}, \forall i=0, \ldots, N$. The step of the mesh is

$$
h=\max _{i=0, \ldots, N} h_{i}
$$

For finite difference methods, we consider

$$
h=h_{i}, \forall i=0, \ldots, N
$$

We have

$$
\begin{gathered}
x_{i+1}=x_{i}+h \text { for any } i=0, \ldots, N, \\
u\left(x_{i}\right) \approx u_{i}, c\left(x_{i}\right) \approx c_{i}, f\left(x_{i}\right) \approx f_{i} \text { et } g\left(x_{i}\right) \approx g_{i} .
\end{gathered}
$$

Thus the problem (3) becomes:

$$
\left\{\begin{array}{l}
-u_{i}^{\prime \prime}+c_{i} u_{i}=f_{i}, \quad \forall \quad i=1, \ldots, N \\
u_{0}^{\prime}=g_{0}-\alpha u_{0}, u_{N+1}^{\prime}=g_{N+1}-\alpha u_{N+1}
\end{array}\right.
$$

Assuming that

$$
u \in C^{2}([0,1])
$$

Using the following

$$
u_{i}^{\prime \prime}=\frac{u_{i+1}-2 u_{i}+u_{i-1}}{h^{2}}
$$

and approximating $u_{0}^{\prime}$ by

$$
\left(\frac{\partial u}{\partial x}\right)_{i} \approx \frac{u_{i+1}-u_{i}}{h}
$$


i.e

$$
u_{0}=\frac{1}{1-\alpha h} u_{1}-\frac{h}{1-\alpha h} g_{0}
$$

and $u_{N+1}^{\prime}$ by

$$
\left(\frac{\partial u}{\partial x}\right)_{i} \approx \frac{u_{i-1}-u_{i}}{h}
$$

i.e

$$
u_{N+1}=\frac{1}{1-\alpha h} u_{N}-\frac{h}{1-\alpha h} g_{N+1}
$$

Then the problem (4) becomes:

$$
\left\{\begin{array}{l}
-\frac{u_{i+1}-2 u_{i}+u_{i-1}}{h^{2}}+c_{i} u_{i}=f_{i} \quad \forall i=0, \ldots, N \\
u_{0}=\frac{1}{1-\alpha h} u_{1}-\frac{h}{1-\alpha h} g_{0} \\
u_{N+1}=\frac{1}{1-\alpha h} u_{N}-\frac{h}{1-\alpha h} u_{N+1}
\end{array}\right.
$$

Therefore (8) may be written in matrix form as

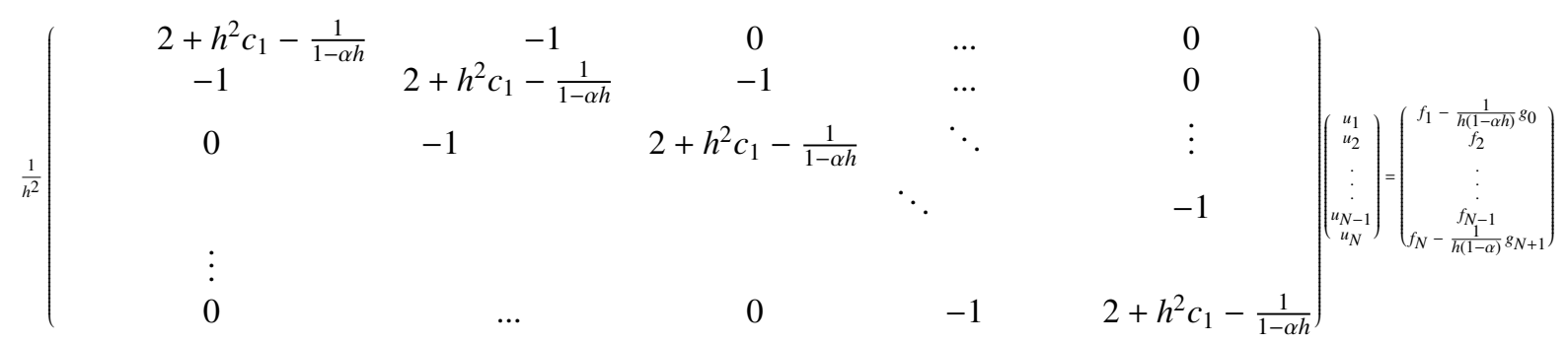

i.e

$$
A_{h} U=B
$$

Let us prove the existence and uniqueness of the solution $u$ of the problem which consist on checking that $A_{h}$ is symmetric positive-definite. i.e

$$
\left\{\begin{array}{l}
A_{h}^{t}=A_{h} \\
\forall v \in \mathbb{R}^{\mathbb{N}},<A_{h} v, v>\geq 0 \\
<A_{h} v, v>=0 \Longrightarrow v=0
\end{array}\right.
$$

Without sacrified the generality, suppose that $\alpha=0$ and assume that $h^{2} c_{i}>1, \quad i=1, \ldots N$.

It's trivial that the matrix $A_{h}$ is symmetric. Choosing $v_{0}=v_{N+1}=0$, we have

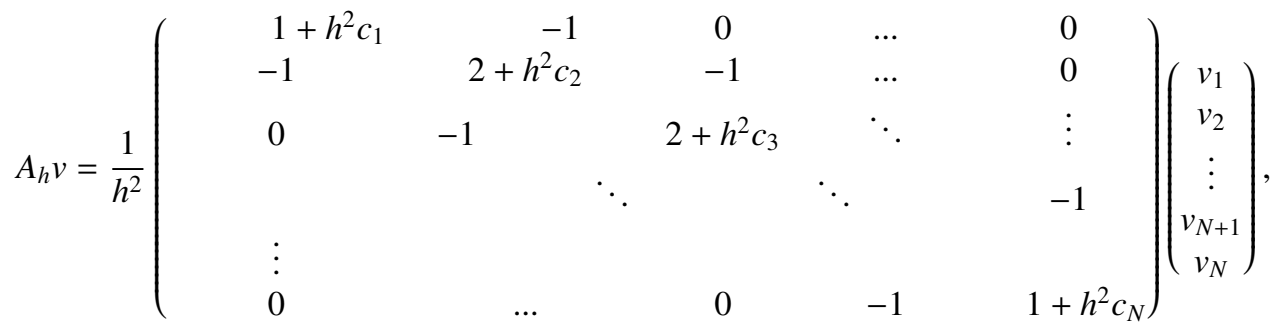

$$
\begin{aligned}
& h^{2}<A_{h} v, v>=\left(\begin{array}{c}
\left(1+h^{2} c_{1}\right) v_{1}-v_{2} \\
-v_{1}+\left(2+h^{2} c_{2}\right) v_{2}-v_{3} \\
-v_{2}+\left(2+h^{2} c_{3}\right) v_{3}-v_{4} \\
\vdots \\
-v_{N-1}+\left(1+h^{2} c_{N}\right)
\end{array}\right)\left(\begin{array}{c}
v_{1} \\
v_{2} \\
\vdots \\
v_{N}
\end{array}\right),
\end{aligned}
$$




$$
\begin{aligned}
& =\left[\left(1+h^{2} c_{1}\right) v_{1}-v_{2}\right] v_{1}+\left[-v_{1}+\left(2+h^{2} c_{2}\right) v_{2}-v_{3}\right] v_{2}+\left[-v_{2}+\left(2+h^{2} c_{3}\right) v_{3}-v_{4}\right] v_{3}+\ldots+\left[-v_{N-1}+\left(1+h^{2} c_{N}\right) v_{N}\right] v_{N}, \\
& =\sum_{i=1}^{N}\left[-v_{i-1}+\left(2+h^{2} c_{i}\right) v_{i}-v_{i+1}\right] v_{i}-v_{N}^{2}-v_{1}^{2}, \\
& =\sum_{i=1}^{N}\left(-v_{i-1} v_{i}\right)+\sum_{i=1}^{N}\left(2 v_{i}^{2}\right)+h^{2} \sum_{i=1}^{N}\left(c_{i} v_{i}^{2}\right)+\sum_{i=1}^{N}\left(-v_{i+1} v_{i}\right)-v_{N}^{2}-v_{1}^{2},
\end{aligned}
$$

Note that

$$
\begin{array}{r}
\sum_{i=1}^{N}\left(-v_{i-1} v_{i}\right)=\sum_{i=2}^{N+1}\left(-v_{i} v_{i-1}\right), \\
\sum_{i=1}^{N}\left(2 v_{i}^{2}\right)=\sum_{i=1}^{N}\left(v_{i}^{2}+v_{i-1}^{2}\right)+v_{N}^{2}
\end{array}
$$

Then it follows that

$$
\begin{aligned}
h^{2}<A_{h} v, v> & =\sum_{i=1}^{N}\left(-2 v_{i} v_{i-1}\right)+\sum_{i=1}^{N}\left(v_{i}^{2}+v_{i-1}^{2}\right)+v_{N}^{2}+h^{2} \sum_{i=1}^{N}\left(c_{i} v_{i}^{2}\right)-v_{N}^{2}-v_{1}^{2}, \\
& =\sum_{i=1}^{N}\left(v_{i}-v_{i-1}\right)^{2}+h^{2} \sum_{i=1}^{N}\left(c_{i} v_{i}^{2}\right)-v_{1}^{2} ; \\
<A_{h} v, v>= & \frac{1}{h^{2}}\left[\sum_{i=1}^{N}\left(v_{i}-v_{i-1}\right)^{2}+h^{2} \sum_{i=2}^{N}\left(c_{i} v_{i}^{2}\right)+\left(h^{2} c_{1}-1\right) v_{1}^{2}\right] \geq 0
\end{aligned}
$$

Hence

$$
\begin{aligned}
<A_{h} v, v>=0 & \Longrightarrow\left\{\begin{array}{l}
v_{i}-v_{i-1}=0 \\
v_{i}=0 \\
\vdots \\
v_{1}=0 .
\end{array}\right. \\
& \Longrightarrow v=0 .
\end{aligned}
$$

Therefore the problem (10) has a unique solution.

\section{Analytical Resolution of the Problem}

In this section we shall use the method of variation of constants to find the exact solution of Robin problem. Let us consider $\quad f(x)=5 \pi^{2} \cos \pi x, g(x)=e^{x}$ and $c(x)=c_{0}$, thus the problem (1) becomes

$$
\left\{\begin{array}{l}
\left.-u^{\prime \prime}(x)+c_{0} u(x)=5 \pi^{2} \cos \pi x, x \in\right] 0,1[ \\
u^{\prime}(0)+\alpha u(0)=1, u^{\prime}(1)+\alpha u(1)=e
\end{array}\right.
$$

The solution of homogeneous equation is

$$
u=k_{1} e^{-\sqrt{c_{o}} x}+k_{2} e^{\sqrt{c_{o}} x}
$$

Now, we seek to find $k_{1}(x)$ and $k_{2}(x)$ such that

$$
u=k_{1}(x) e^{-\sqrt{c_{o}} x}+k_{2}(x) e^{\sqrt{c_{o}} x}
$$

satisfies the first equation of (11). Then we have to solve the following system of equations

$$
\left\{\begin{array}{l}
k_{1}^{\prime}(x) e^{-\sqrt{c_{o}} x}+k_{2}^{\prime}(x) e^{\sqrt{c_{o}} x}=0 \\
-\sqrt{c_{o}} k_{1}^{\prime}(x) e^{-\sqrt{c_{o}} x}+\sqrt{c_{o}} k_{2}^{\prime}(x) e^{\sqrt{c_{o}} x}=-5 \pi^{2} \cos \pi x
\end{array}\right.
$$

We obtain 


$$
\begin{aligned}
& k_{1}^{\prime}(x)=\frac{5}{2 \sqrt{c_{o}}} \pi^{2} \cdot e^{\sqrt{c_{o}} x} \cdot \cos \pi x \\
& k_{2}^{\prime}(x)=-\frac{5}{2 \sqrt{c_{o}}} \pi^{2} \cdot e^{-\sqrt{c_{o}} x} \cos \pi x .
\end{aligned}
$$

i.e

$$
k_{1}(x)=\frac{5}{2 \sqrt{c_{o}}} \pi^{2} \int e^{\sqrt{c_{o}} x} \cos \pi x d x
$$

and

$$
k_{2}(x)=-\frac{5}{2 \sqrt{c_{o}}} \pi^{2} \int e^{-\sqrt{c_{o}} x} \cos \pi x d x .
$$

Integrating twice by parts we obtain

$$
\begin{gathered}
k_{1}(x)=\frac{5 \pi^{3}}{2 \sqrt{c_{o}}\left(\pi^{2}+c_{o}\right)} e^{\sqrt{c_{o} x}} \sin \pi x+\frac{5 \pi^{2}}{2\left(\pi^{2}+c_{o}\right)} e^{\sqrt{c_{o}} x} \cos \pi x+\lambda, \\
k_{2}(x)=\frac{-5 \pi^{3}}{2 \sqrt{c_{o}}\left(\pi^{2}+c_{o}\right)} e^{-\sqrt{c_{o}} x} \sin \pi x+\frac{5 \pi^{2}}{2\left(\pi^{2}+c_{o}\right)} e^{-\sqrt{c_{o}} x} \cos \pi x+\beta .
\end{gathered}
$$

Then, the relation (12) becomes:

$$
u=\frac{5 \pi^{2}}{\pi^{2}+c_{o}} \cos \pi x+\lambda e^{-\sqrt{c_{o}} x}+\beta e^{\sqrt{c_{o}} x}
$$

Let us find $\lambda$ and $\beta$ of (14). By using the boundary conditions of the problem (11), it follows that

$$
\left\{\begin{array}{l}
\left(\alpha-\sqrt{c_{o}}\right) \lambda+\left(\alpha+\sqrt{c_{o}}\right) \beta=1-\frac{5 \pi^{2} \alpha}{\pi^{2}+c_{o}} \\
\left(\alpha-\sqrt{c_{o}}\right) e^{-\sqrt{c_{o}}} \cdot \lambda+\left(\alpha+\sqrt{c_{o}}\right) e^{\sqrt{c_{o}}} \cdot \beta=e+\frac{5 \pi^{2} \alpha}{\pi^{2}+c_{o}}
\end{array}\right.
$$

We then obtain

$$
\begin{aligned}
& \lambda=\frac{e-e^{\sqrt{c_{o}}}+\left(1+e^{\sqrt{c_{o}}}\right)\left(\frac{5 \pi^{2} \alpha}{\pi^{2}+c_{o}}\right)}{-2 \alpha \sinh \sqrt{c_{o}}+2 \sqrt{c_{o}} \sinh \sqrt{c_{o}}} \\
& \beta=\frac{e-e^{-\sqrt{c_{o}}}+\left(1+e^{-\sqrt{c_{o}}}\right)\left(\frac{5 \pi^{2} \alpha}{\pi^{2}+c_{o}}\right)}{2 \alpha \sinh \sqrt{c_{o}}+2 \sqrt{c_{o}} \sinh \sqrt{c_{o}}}
\end{aligned}
$$

The relation (14) becomes

$$
\begin{gathered}
u=\frac{5 \pi^{2}}{\pi^{2}+c_{o}} \cos \pi x+\frac{\left(e-e^{\sqrt{c_{o}}}\right) e^{-\sqrt{c_{o}} x}+\left(1+e^{\sqrt{c_{o}}}\right)\left(\frac{5 \pi^{2} \alpha}{\pi^{2}+c_{o}}\right) e^{-\sqrt{c_{o}} x}}{2 \sinh \sqrt{c_{o}}\left(\sqrt{c_{o}}-\alpha\right)} \\
+\frac{\left(e-e^{-\sqrt{c_{o}}}\right) e^{\sqrt{c_{o}} x}+\left(1+e^{-\sqrt{c_{o}}}\right)\left(\frac{5 \pi^{2} \alpha}{\pi^{2}+c_{o}}\right) e^{\sqrt{c_{o}} x}}{2 \sinh \sqrt{c_{o}}\left(\sqrt{c_{o}}+\alpha\right)}, \\
u=\frac{5 \pi^{2}}{\pi^{2}+c_{o}} \cos \pi x+Q .
\end{gathered}
$$

With

$$
\begin{aligned}
& \text { With } \\
& \qquad \begin{array}{l}
Q=\frac{\left(e-e^{\sqrt{c_{o}}}\right) e^{-\sqrt{c_{o}} x}+\left(1+e^{\sqrt{c_{o}}}\right)\left(\frac{5 \pi^{2} \alpha}{\pi^{2}+c_{o}}\right) e^{-\sqrt{c_{o}} x}}{2 \sinh \sqrt{c_{o}}\left(\sqrt{c_{o}}-\alpha\right)}+\frac{\left(e-e^{-\sqrt{c_{o}}}\right) e^{\sqrt{c_{o}} x}+\left(1+e^{-\sqrt{c_{o}}}\right)\left(\frac{5 \pi^{2} \alpha}{\pi^{2}+c_{o}}\right) e^{\sqrt{c_{o}} x}}{2 \sinh \sqrt{c_{o}}\left(\sqrt{c_{o}}+\alpha\right)} ; \\
=\frac{1}{2 \sinh \sqrt{c_{o}}}\left[\frac{\left(e-e^{\sqrt{c_{o}}}\right) e^{-\sqrt{c_{o}} x}+\left(1+e^{\sqrt{c_{o}}}\right)\left(\frac{5 \pi^{2} \alpha}{\pi^{2}+c_{o}}\right) e^{-\sqrt{c_{o}} x}}{\sqrt{c_{o}}-\alpha}+\frac{\left(e-e^{-\sqrt{c_{o}}}\right) e^{\sqrt{c_{o}} x}+\left(1+e^{-\sqrt{c_{o}}}\right)\left(\frac{5 \pi^{2} \alpha}{\pi^{2}+c_{o}}\right) e^{\sqrt{c_{o}} x}}{\sqrt{c_{o}}+\alpha}\right] \\
=\frac{1}{2 \sinh \sqrt{c_{o}}\left(c_{o}+\alpha^{2}\right)}\left[2 e \sqrt{c_{o}} \cosh \sqrt{c_{o}} x-2 \sqrt{c_{o}} \cosh \left(\sqrt{c_{o}}-\sqrt{c_{o}} x\right)\right.
\end{array}
\end{aligned}
$$




$$
\begin{aligned}
& +2 \sqrt{c_{o}}\left(\cosh \sqrt{c_{o}} x+\cosh \left(\sqrt{c_{o}}-\sqrt{c_{o}} x\right)\right) \frac{5 \pi^{2} \alpha}{\pi^{2}+c_{o}}-2 e \alpha \sinh \sqrt{c_{o}} x-2 \alpha \sinh \left(\sqrt{c_{o}}-\sqrt{c_{o}} x\right)+ \\
& \left.-2 \alpha\left(\sinh \sqrt{c_{o}} x-\sinh \left(\sqrt{c_{o}}-\sqrt{c_{o}} x\right)\right) \frac{5 \pi^{2} \alpha}{\pi^{2}+c_{o}}\right]
\end{aligned}
$$

Therefore the exact solution of (11) can be written in the form

$u=\frac{5 \pi^{2}}{\pi^{2}+c_{o}} \cos \pi x+\frac{\left(e+\frac{5 \pi^{2} \alpha}{\pi^{2}+c_{o}}\right)\left(\sqrt{c_{o}} \cosh \sqrt{c_{o}} x-\alpha \sinh \sqrt{c_{o}} x\right)+\left(\frac{5 \pi^{2} \alpha}{\pi^{2}+c_{o}}-1\right)\left(\sqrt{c_{o}} \cosh \left(\sqrt{c_{o}}-\sqrt{c_{o}} x\right)+\alpha \sinh \left(\sqrt{c_{o}}-\sqrt{c_{o}} x\right)\right)}{\sinh \sqrt{c_{o}}\left(c_{o}+\alpha^{2}\right)}$

Taking $\alpha=0$, we have therefore

$$
u(x)=\frac{5 \pi^{2}}{\pi^{2}+c_{o}} \cos \pi x+\frac{e \sqrt{c_{0}} \cosh \sqrt{c_{0}} x-\sqrt{c_{O}} \cosh \left(\sqrt{c_{0}}-\sqrt{c_{0}} x\right)}{c_{0} \sinh \sqrt{c_{0}}}
$$

\section{Numerical Simulations}

The aim here is to represent on the same graph the solutions (18) and (10) exact and numerical, respectively, taking into account the number of points $N$ and the step $h$ of the finite difference method in order to converge the two solutions. This simulation will be implemented in Scilab.

Figure 1 illustrates the exact solution (18) for $c_{0}=3$.

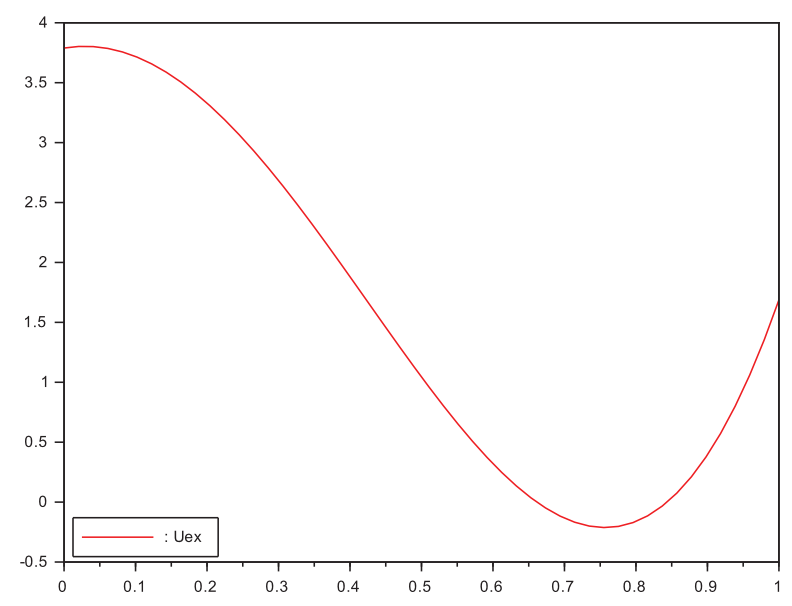

Figure 1. Representation of the exact solution

By fixing $N=5$ in (10), we have attempted to vary the step $h$ of the method to verify the numerical convergence of numerical solution of (10) on the exact solution of (18). ( See Figure 2).

- Taking $h=0.001$, we notice that both exact and numerical solutions converge almost everywhere numerically ( See Figure 2 a ).

- Taking $h=0.01$, we find that at the beginning the two solutions tend to go away and at some point converge and then move away very quickly, which is explained by slow numerical convergence ( See Figure $2 \mathrm{~b}$ ).

- Taking $h=0.5$, we notice that the exact and numerical solutions, respectively, have almost a tendency not to converge except on a few points, this observation can be explained by a numerical divergence ( See Figure $2 \mathrm{c}$ ). 


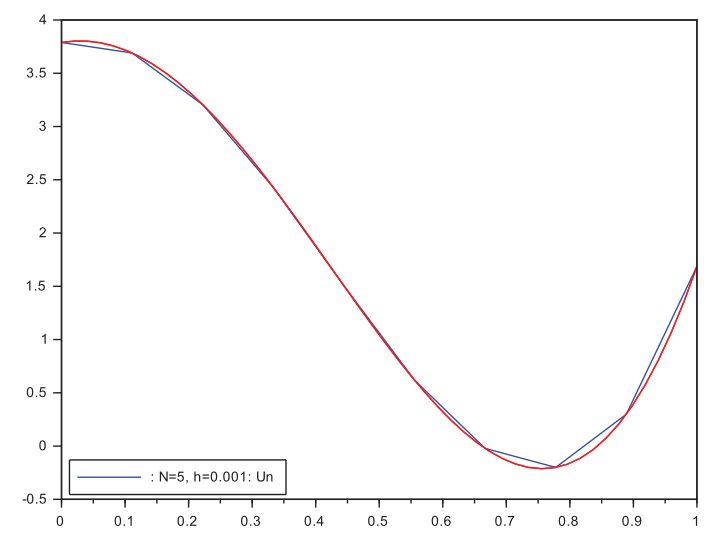

(a)

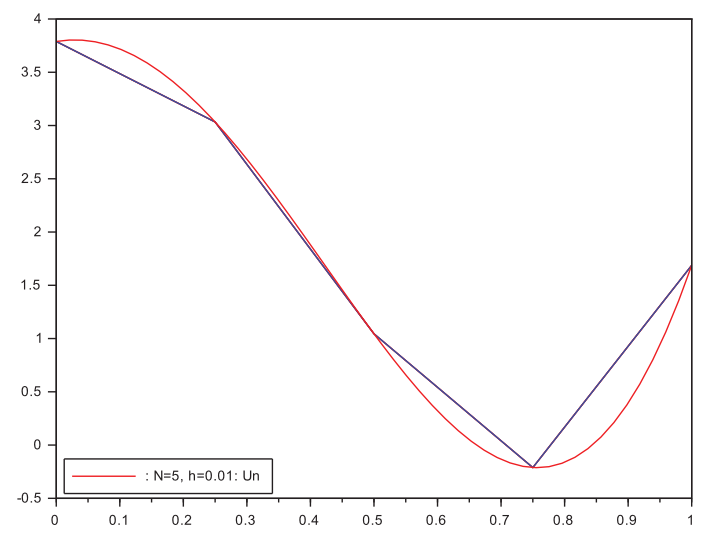

(b)

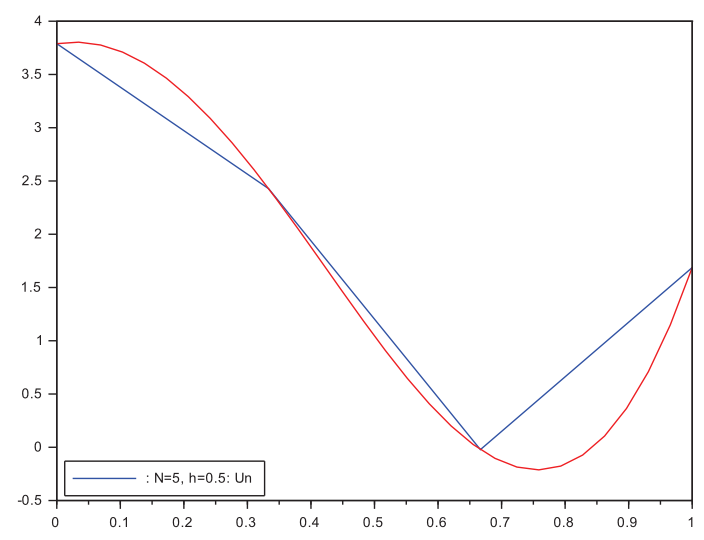

(c)

Figure 2. Representation of the exact and numerical solutions for $N=5$

In the Figure 3, we fixed $N=10$.

- Taking $h=0.001$ and $h=0.01$, we observe a very fast numerical convergence for both exact and numerical solutions ( See Figure 3 d and Figure 3 e).

- Taking $h=0.5$, we notice that the exact and numerical solutions, respectively, converge numerically almost everywhere. ( See Figure 3 f) 


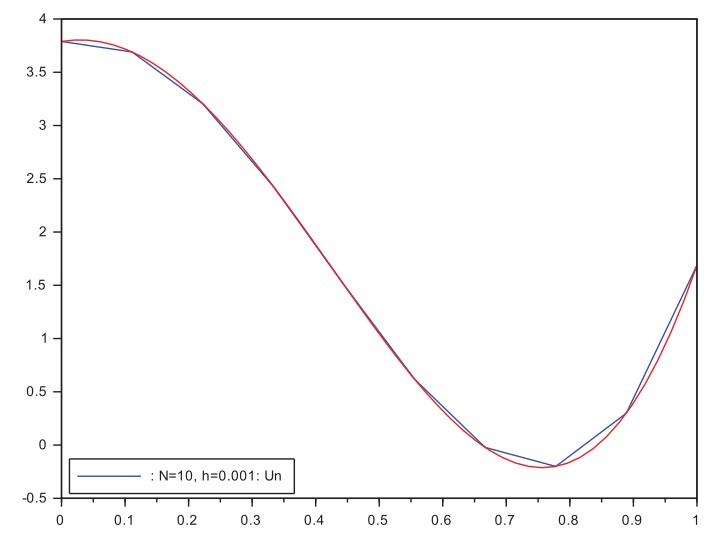

(d)

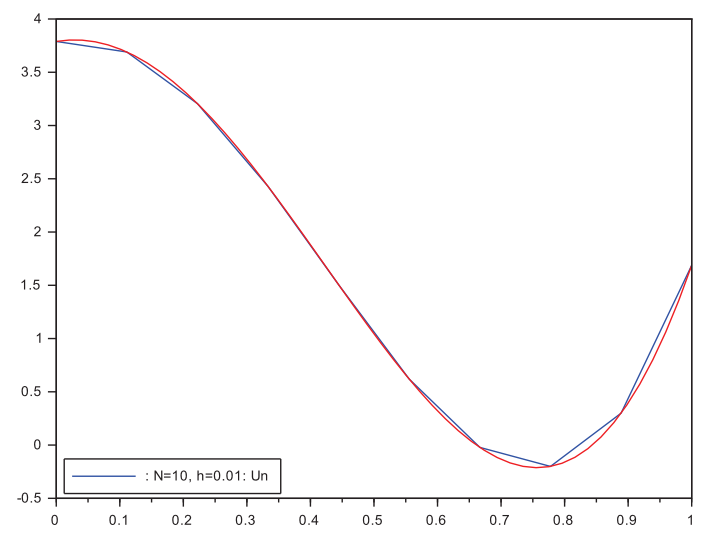

(e)

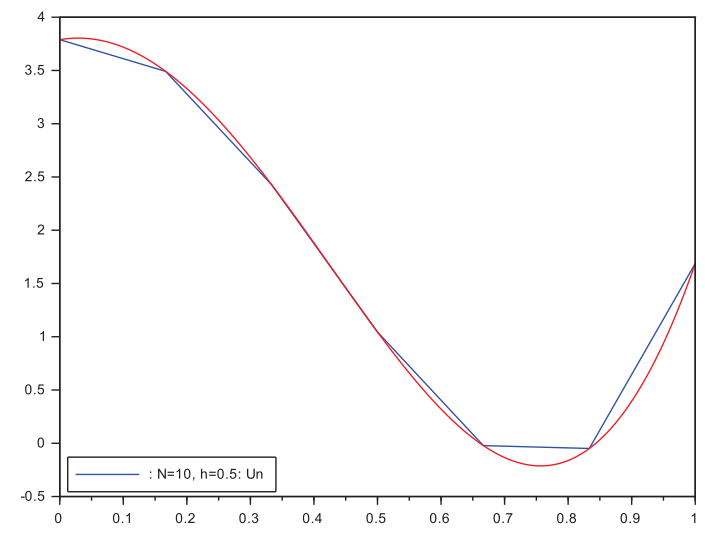

(f)

Figure 3. Representation of the exact and numerical solutions for $N=10$

The finite difference method being linked to a multiplicative term of the form $\frac{1}{h^{2}}$, requires a very large number of points $N$ and a very great choice of the step $h$ of the method to ensure the convergence of the numerical solution towards the exact solution.

\section{Conclusion and Perspectives}

The numerical resolution of PDEs remains a challenge. In this paper, the existence and uniqueness of the solution of Robin's problem has been demonstrated by Lax-Milgram theorem. In order to find the numercial solution of the problem we have used the finite difference method. In fact, in order to prove the existence and uniqueness of the solution of the related linear system, it was appropriate to impose $\alpha=0$ and $h^{2} c_{i}>1, \quad i=1, \ldots, N$. We have employed the method of variation of constants to find the exact solution. Finally, we have implemented numerical simulation in $S$ cilab to approach the numerical solution towards the exact solution.

In the future we will inverstigate the theoretical convergence of this model. Furthermore, we will try to solve the two or three-dimensional Robin's problem.

\section{References}

Aslak, T., \& Ragnar, W. (2005). Introduction to partial differential equations: a computational approach. SpringerVerlag. https://doi.org/10.1007/b138016

Beghr, H., \& Harutyunyan, G. (2006). Robin boundary value problem for the Poisson equation. J. Anal. Appl., 4, $201-213$. 
Beghr, H., Saule, B., \& Harutjunjan. G. (2017). Remark on Robin problem for Poisson equation. Complex Variables and $\begin{array}{lllll}\text { Elliptic Equations } & \text { An International } & \text { Journal, } & 62, & 1589-1599 .\end{array}$ https://doi.org/10.1080/17476933.2017.1303052

Bourchra, B., Guillaume, C., \& Jacques, L. (2006). Penalization of Robin Boundary condition. Applied Numerical Mathematics, 96, 134-152. https://doi.org/10.1016/j.apnum.2015.06.001

Dagmar, M. (1998). Solution of the Robin problem for the Laplace equation. Applications of Mathematics, 43, 133-155. https://doi.org/10.1023/A:1023267018214

Daniel, D. (2000). Robin Boundary Value Problems on Arbitrary Domains. Transactions of the American Mathematical Society, 9, 4207-4236. https://doi.org/10.1090/S0002-9947-00-02444-2

Gabriel, A., Maria, G. A., Ricardo, G. D., \&Ariel, L. L. (2015). Nonhomogeneous Neumann problem for the Poisson equation in domains with an external cusp. J. Math. Anal. Appl, 310, 397-411. https://doi:10.1016/j.jmaa.2005.01.065

Hinze, M., Pinnau, R., Ulbrich, M., \& Ulbrich. S. (2009). Mathematical modelling: Theory and Applications. Springer. https://doi.org/ 10.1007/978-1-4020-8839-1

John, C. S. (2004). Finite Difference Schemes and Partial Differential Equations (2nd ed.) Siam. https://doi.org/10.1137/1.9780898717938

Loredana, L., \& Osvaldo, M. (2006). The Poisson's problem for the Laplacian with Robin boundary condition in nonsmooth domains. Rev. Math. Iberoamericana, 22, 181-204. https://doi.org/10.4171/RMI/453

Peter, K., \& Lutz, A. (2000). Numerical methods for elliptic and parabolic partial differential equations (2nd ed.) Springer. https://doi.org/ 10.1007/b97419

Shao-Gao, D. (2009). Positive solutions for Robin problem involving the p(x)-Laplacian. J. Math. Anal. Appl., 360,548560. https://doi.org/10.1016/j.jmaa.2009.06.032

Thomas, J. W. (1995). Numerical Partial Diffential Equations: Finite Difference Methods. Springer. https://doi.org/10.1007/978-1-4899-7278

\section{Copyrights}

Copyright for this article is retained by the author(s), with first publication rights granted to the journal.

This is an open-access article distributed under the terms and conditions of the Creative Commons Attribution license (http://creativecommons.org/licenses/by/4.0/). 